Cuadernos de Filología Italiana

ISSN: 1133-9527

https://dx.doi.org/10.5209/cfit.62542

\title{
Il genio e l'ingegno. Storia e statuto del romanzo secondo Rovani
}

\author{
Alejandro Patat ${ }^{1}$
}

Ricevuto: 27 novembre 2018 / Modificato: 10 maggio 2019 / Accettato: 28 giugno 2019

Riassunto: L'articolo si propone di affrontare le riflessioni estetiche di Giuseppe Rovani, focalizzando la storia e lo statuto del romanzo. A tal fine, risulta imprescindibile fare una lettura trasversale della critica dell'opera di Rovani per capire quali siano stati i punti nodali della messa in discussione, delle stroncature e della riabilitazione dei Cento anni, considerato unanimemente il suo capolavoro. L'analisi delle sue posizioni teoriche attorno al romanzo nell'Ottocento italiano permette, quindi, di capire meglio quali saranno le nuove esplorazioni in campo narrativo una volta tramontato il modello manzoniano.

Parole chiave: Rovani, Manzoni, romanzo, estetica, critica.

\section{[en] Genio and ingegno. History and status of the novel according to Rovani}

\begin{abstract}
The article aims to address the aesthetic reflections of Giuseppe Rovani, which focus on the story and the status of the novel. For this purpose, it is essential to make a cross-reading of the criticism of the work of Rovani in order to understand what the nodal points of the crutch and rehabilitation of Cento Anni, unanimously considered his masterpiece, were. The analysis of his theoretical positions around the Italian novel in the nineteenth century allows, therefore, to better understand what the new explorations in the narrative field will be, once the Manzoni model has been set.
\end{abstract}

Keywords: Rovani, Manzoni, novel, aesthetics, critics.

Sommario: 1. Centocinquant'anni di critica rovaniana 1.1 Cento anni di pregiudizi e stroncature 1.2. Capire Rovani: gli studi recenti di Baldi e Tamiozzo Goldman 2. Storia e statuto del romanzo 2.1. Metodo di indagine 2.2. Canone ottocentesco 2.3 Concetti e categorie 3. Per finire.

Come citare: Patat, Alejando (2019): «Il genio e l'ingegno. Storia e statuto del romanzo secondo Rovani», Cuadernos de Filología Italiana, 26, pp. 197-220.

\section{Centocinquant'anni di critica rovaniana}

\subsection{Cento anni di pregiudizi e stroncature}

Giuseppe Rovani ha scritto sei romanzi: Lamberto Malatesta (1843), Valenzia Candiano (1844), Manfredo Pallavicino (1845-1846), Cento anni (1859-1864, seconda edizione definitiva: 1868-1869), La Libia d'oro (1868) e La giovinezza di

\footnotetext{
1 Università per Stranieri di Siena. Dipartimento d'Ateneo per la Didattica e la Ricerca. P.zza Carlo Rosselli 27/28 (5300) Siena. patat@unistrasi.it
} 
Giulio Cesare (1872-1873). Si tratta di trent'anni quasi ininterrotti di produzione narrativa, cui si aggiungono le riflessioni estetiche, prima raccolte nel IV volume della Storia delle Lettere e delle Arti in Italia (1858), ristampato con il titolo di Biografie dei più celebri italiani del secolo decimonono (1865) e che verrà pubblicato postumo, con qualche saggio in più, frutto della sua attività giornalistica, come Le Tre Arti (1874). In esso Rovani affronta la storia e lo statuto delle arti nell'Ottocento italiano, introducendo concetti e categorie che illuminano, a nostro avviso, il suo stesso progetto creativo. L'autore milanese ha tentato anche la strada del teatro e della storia, ma non c'è alcun dubbio che la sua maggiore preoccupazione da "artista" - come egli stesso si è tante volte definito - sia ruotata attorno al romanzo.

La critica della narrativa rovaniana segue due filoni principali: "l'agiografia" in chiave scapigliata della Rovaniana (Dossi 1946) o la messa in discussione in ambito storico-letterario ed estetico propiziata da Croce (1914). Nel capitolo «Giuseppe Rovani - Ippolito Nievo», alla luce ormai delle riflessioni che lo hanno portato all'esercizio critico che distingue la poesia dalla non poesia, Croce accomuna Rovani e Nievo, in quanto autori che si occupano della rappresentazione di uno stesso periodo della storia. «E strano è, soprattutto, parlare di novità e di originalità, quando mi pare che basti volgere uno sguardo ai romanzi di Rovani per ravvivare subito in lui nient'altro che un manzoniano. Un manzoniano della prima epoca, anteriore cioè al discorso sul romanzo storico» (Croce 1914: 112).

Tale giudizio non riguarda solo i romanzi di Rovani, ma si fa estensivo a Le Tre Arti, del quale Croce riferisce, non senza ironia, che secondo Dossi esso conterrebbe «tesori di critica» (Croce 1914: 112). Sappiamo già che, negli stessi anni in cui Cento anni usciva a puntate sulla Gazzetta di Milano, Rovani rispondeva puntualente alle critiche che gli muovevano i suoi detrattori. Ma le parole di Croce rappresentano invece la prima messa in discussione della critica ufficiale che, anche se esplicitamente relativa ai romanzi, abbraccia implicitamente la saggistica. Manzoniana sarebbe la tendenza alla composizione mista di storia ed invenzione e «manzoniani sono altresì gli accorgimenti ai quali il narratore ricorre, ora per rendere accettabile la sua storia al lettore, ora per allontanare da lui certe brame, che egli non può soddisfare» (Croce 1914: 113). «Manzoniani sono, infine, e comuni alla scuola lombarda, la riflessione morale e politica e l'intento ammonitivo ed educativo dei suoi romanzi» (Croce 1914: 114).

Mentre la prima osservazione di Croce, quella dell'adesione di Rovani al romanzo storico, è stata definitivamente ridiscussa dalla critica nella tesi di Baldi sull'erosione del romanzo storico da parte dell'autore milanese (Baldi 1967), la seconda osservazione, effettivamente, sta ad indicare che se c'è una lezione manzoniana assimilata da Rovani, essa consiste nella centralità del lettore come cooperatore del testo, secondo le note parole di Umberto Eco (1979: 50-66). Infine, la terza osservazione, che apre una serie molto ricca d'interventi - in particolare, come vedremo, nel noto allievo della scuola storica Guido Mazzoni (1938) -, ha bisogno però di ulteriori conferme o precisazioni.

«Ma ciò che a Rovani manca del Manzoni è l'ideale determinato e fortemente sentito e la capacità di rappresentarlo in figure artistiche» (Croce 1914: 114). L'affondo primario della critica crociana darà luogo a sua volta a due filoni ben distinti: da una parte, l'attacco frontale nei confronti della figura di Rovani come autore, debole nella sua intenzione morale e civile, da cui non è esclusa la sua vicenda biogra- 
fica; dall'altra, la stroncatura più specifica della resa stilistica della sua opera, intesa come testimonianza incompiuta di un tentativo artistico non riuscito².

Il primo filone, inaugurato da Croce, ha inoltre una sfumatura significativa: Rovani sarebbe scevro di spinte etico-civili comparativamente rispetto a Nievo, al punto che, per il critico napoletano, Nievo sarebbe «il vero prosecutore di Manzoni, cioè innovatore: in quanto elabora e propone una concezione della vita e un ideale morale» (Croce 1914: 116). La sfumatura sta nel fatto che da questo momento in poi l'esercizio critico su Rovani dovrà fare i conti con la letteratura nieviana, ora avvicinando ora allontanando i loro rispettivi capolavori. Ciononostante, pur facendo un accenno indiretto alla teoria dell'arte in Rovani, Croce, secondo una prospettiva cara all'approccio storico-letterario a partire da De Sanctis, ne centra il problema chiave, cioè, la necessaria solidità di un progetto narrativo per potersi considerare prosecutore e, quindi, innovatore nei confronti dell'immediato antecessore.

Neanche Guido Mazzoni si distacca molto dalla critica crociana, nonostante riconosca dei meriti ai Cento anni. Intanto, il suo affresco panoramico sull'Ottocento esclude Rovani dal capitolo interamente dedicato agli scritti di propaganda civile e politica, dove vengono esaminati puntualmente sia i periodici dell'epoca, dal Politecnico al Viesseux, dal Crepuscolo al Pungolo, sia i contributi di una serie molto articolata di autori che avrebbero costruito un dibattito politico-sociale, etico-civile, artistico-culturale tra gli anni ' 40 e '60: Tenca, Camerini, Mazzini, Cattaneo, Correnti, Gioberti, Balbo, Tommaseo, D'Azeglio, Ferrari, Mamiani e Settembrini. Nessuna menzione alla prosa giornalistica di Rovani, né alle sue incursioni nella critica letteraria, teatrale e d'arte sui più importanti giornali milanesi dell'epoca. Esclusione di Rovani che si estende anche ai capitoli relativi al teatro, ai filosofi, storici e critici dell'Ottocento.

L'opera narrativa di Rovani, invece, viene inserita da Mazzoni all'interno del capitolo sul romanzo storico. Rispetto al primo filone crociano, quello sulla fragilità morale dell'autore, la sua lettura rimane invariata. E l'accenno a Nievo dimostra quanto il binomio creato da Croce sia funzionale al gioco del saliscendi, il cui equilibrio consiste perlopiù nell'innalzare l'autore delle Confessioni e nell'affondare quello dei Cento anni: «il suo intento non è così direttamente patriottico come quello delle Confessioni〉 (Mazzoni 1938: 906).

L'attaco da tanta parte della critica sull'Ottocento italiano, imperniata sulla ricostruzione storica e storicistica dei valori risorgimentali, giudica Rovani come un funambulo che cammina irriverente sulla fune austriaca al di sopra del terreno stabile della stessa tradizione italiana. Distratta appare, però, tale critica, dal fatto che i famigerati scritti di Rovani sulla visita degli Imperatori d'Austria in Italia nel 1856 non sono altro che una celebrazione "patriottica" del primato della cultura italiana tout court $^{3}$. Mazzoni è schietto (1938: 906-907): «la sua salute guastò con l'assenzio; peggio a sé stesso aveva nociuto quando egli, già volontario per l'indipendenza, si diede all'Austria». A niente era valsa, quindi, l'appassionata difesa di Beniamino Gutierrez (1934-1935) ne «La tragedia spirituale di Giuseppe Rovani», introduzione

2 Anche se alla fine del suo intervento, Croce (1914: 115) sembra recuperare l'ultimo romanzo di Rovani, $L a$ giovinezza di Giulio Cesare: «lo stile, da discorsivo che era nei Cento Anni, si viene mutando in poetico». La conclusione del suo saggio è però netta: «lascia l'impressione di uno sforzo non riuscito» (Croce 1914: 116).

3 Com'è noto, dal 13 gennaio al 6 marzo 1857 Rovani scrisse sulla Gazzetta di Milano ventotto articoli giornalistici sulla visita imperiale austriaca a Milano. Il fatto gli valse la censura dei patrioti ma anche degli amici del suo cenacolo e compromise per sempre la sua reputazione civile. 
al commento, anch'esso partigiano, del romanzo ciclico. A niente sarebbe valsa, inoltre, la versione agiografica della generazione scapigliata.

L'offensiva etico-politica contro Rovani non impedisce a Mazzoni (1938: 906) di considerare i Cento anni un libro che «si legge con piacere» e che «procede sempre attraente per chi si diletta delle cronistorie e vi ritrova immagini note o utile complemento di cognizioni» così come «sono innegabili l'abilità di raggruppare tanti personaggi e tante vicende attorno ad un processo, la brava esecuzione di ritratti fedelmente schizzati, l'acuta percezione di alcuni affetti» (Mazzoni 1938: 906). In sintesi, nessun accenno al Rovani critico e qualche riconoscimento parziale al romanzo, che Mazzoni riduce a mera esecuzione pittorescca di tavole d'epoca, che, più che storiche, si addicono alla cronistoria, implicitamente intesa come surrogato della storia.

Come si vede, con gli anni s'intensifica il secondo filone, quello che riguarda il merito stesso della sua opera e, in particolare, dei Cento anni. A suggellare la stroncatura, in aiuto della larga schiera di denigratori dell'opera rovaniana, viene Aldo Borlenghi (1961: xxix), per il quale tutto l'apparato concettuale dello scrittore milanese, dagli studi sull'arte e sulla storia, dalle conoscenze delle scienze naturali alla medicina sono «in generale ad orecchio» e persino due testi in genere meno attaccati come La Libia d'oro e La giovinezza di Giulio Cesare, sottolineano «l'esteriore provvisorietà e la scarsa incidenza di ogni ricerca» (Borlenghi 1961: xxx). Nella visione d'insieme poi, anche il lavoro critico di Rovani rientra nella sfera delle «attività provvisorie, rette da un'informazione appena giornalistica, e dalla necessità di seguire, più che l'arte, le forme di spettacolo in quanto elementi della storia del costume» (Borlenghi 1961: xxix). Rimane chiaro quanto per Borlenghi un romanzo con ambizioni storiche debba essere supportato da una competenza da storiografo e quanto i generi non possano avere tutti lo stesso statuto. Quindi, l'interesse di Rovani per il mondo teatrale (che lui, in modo davvero originale, presenta dalle quinte) non risponderebbe ad una alta visione organica della produzione artistica. Infine, causa della negligenza rovaniana sarebbe stato «lo scotto di aver rinunciato alla suggestione del modello manzoniano» (Borlenghi 1961: xxx). Notevole, però, che quest'affermazione in negativo, buttata là, in mezzo ad una critica dispregiativa dell'opera rovaniana, abbia saputo cogliere nel segno di una scelta estetica di Rovani: aver rinunciato a Manzoni.

\subsection{Capire Rovani: gli studi recenti di Baldi e Tamiozzo Goldmann}

Come si sa, il primo intervento critico di riabilitazione di Rovani "nel merito" lo dobbiamo a Baldi, spinto forse dalla allora recente pubblicazione della Rovaniana, che offriva nuovi materiali per l'indagine e dell'autore e dell'opera. «Gli inizi dell'iter narrativo [di Rovani] si situano ancora decisamente, seppur in maniera alquanto epigonica, nell'area del romanzo storico proliferato sul tronco scottiano-manzoniano» (Baldi 1967: 7). Questa frase ci potrebbe insospettire e indurre a pensare preventivamente che il libro di Baldi si orienti di nuovo verso quella critica della comparazione, che ha stigmatizzato (e pure necrotizzato) Rovani nel segno di un'assenza, di una mancanza, di un'impossibilità. Invece, secondo Baldi (1967: 7), contro questa prima maniera e, in particolare contro il romanzo storico, Rovani «assumerà, in anni più maturi, un contegno fortemente polemico». A dire la verità, la linea di lettura, attenta al carattere evolutivo dell'opera rovaniana, tiene conto di tutto ciò che lo scrittore milanese stesso ha esposto ne Le Tre Arti sul Castello di Trezzo, su Marghe- 
rita Pusterla, su Ettore Fieramosca, testi a partire dai quali Rovani avrebbe messo in evidenza lo spegnersi progressivo del romanzo storico. La polemica di Rovani è contro 1'atmosfera erotico-sentimentale del genere tra gli anni '30 e '40 e contro il «protendersi nostalgico verso un altrove temporale» (Baldi 1967: 11). L'ipotesi di Baldi è che, erodendo il romanzo storico, Rovani aspiri a qualcos'altro all'interno del sistema narrativo, qualcosa di diverso e di originale.

Si profila, dunque, l'idea di un romanzo che non sia solo regressione temporale idealizzante né puro intreccio, né libello edificante, bensì un territorio più vasto d'indagine politica, storica, sociale e culturale. Baldi è il primo a considerare la Rovania$n a$ e, in particolare i frammenti espunti dalla prima e dalla seconda edizione in volume dei Cento anni, scritti originariamente per l'edizione a puntate sulla Gazzetta di Milano, un vero laboratorio di germinazione del romanzo. Nello stesso tempo, i rimandi a Le Tre Arti sono per Baldi riflessioni critiche fondamentali e punto di appoggio teorico per l'elaborazione del testo. I Cento anni sarebbero alla fine «la summa della cultura e dell'esperienza dell'autore, l'ideale luogo geometrico di tutti i suoi interessi» (Baldi 1967: 66). Per la prima volta, fuori dall'agiografia di Gutierrez e dall'egida dossiana, un critico afferma - anche se in nota, cioè al margine del corpo del proprio discorso critico - che «Rovani s'inserisce appieno nella direzione di sviluppo del romanzo ottocentesco» (Baldi 1967: 73, n. 1).

La congiunzione tra passato e presente, la convergenza tra saggio e narrativa comportano una svolta del romanzo verso un nuovo orizzonte e lo trasformano più in strumento d'indagine che in puro divertimento. Baldi sarebbe il primo a contestare l'accusa della critica contro l'architettura "imperfetta" dei Cento anni, perché misto di fantasia e "digressioni" o perché costruito sulla base di un intreccio debole o perché senza una fitta rete di personaggi ben definiti. Per Baldi (1967: 97), giustamente, tale critica «è priva di fondamento dal punto di vista storico, perché è componente fondamentale del romanzo europeo moderno l'esigenza di arricchire la fabula con una gamma di molteplici interessi conoscitivi».

Paradossalmente, guadagnato tanto terreno perso, alla fine la critica di Baldi (1967: 101) non si discosta molto da quella dei predecessori:

Ed in effetti è doveroso riconoscere che i Cento anni sono rimasti lontani dalla loro immagine ideale. Il quadro grandioso di un'epoca intera, esplorato in tutti i suoi aspetti, la vita pubblica, il pensiero e le arti, il costume stenta a delinearsi dinanzi agli occhi del lettore; le varie componenti non riescono ad articolarsi in un insieme unitario, che dia l'impressione della complessità e della organicità del processo storico, ma sono allineate l'una affianco dell'altra, con procedimento un po' meccanico, all'uso delle voci di un'enciclopedia. Anche la qualità intrinseca dei numerosi inserti saggistici non è di altissimo livello, poiché manca a Rovani la necessaria vastità e profondità di cultura (a cui egli cerca di supplire con una minuta e ghiotta erudizione, animata da uno spirito mordace e brillante); ma soprattutto manca quel lucido rigore intellettuale, quella profonda sostanza etica ed umana che costituiscono il fascino delle digressioni di carattere storico ed economico dei Promessi Sposi e le rendono così vive e stimolanti per l'intelligenza del lettore.

Come si vede, dopo aver confutato una ad una le posizioni della critica nei confronti di Rovani per due motivi - aver avuto una idea pregressa di romanzo sulla quale giudicare i suoi e aver letto tutto alla luce della produzione manzoniana-, 
Baldi incorre sorprendentemente nella stessa strategia valutativa. Non solo è contestabile il suo giudizio negativo sul basso livello degli inserti saggistici o sulla cultura scarsa di Rovani (davvero stupefacente questo giudizio verso uno che aveva dato luogo ad una compilazione della storia delle lettere italiane, avendo raccolto i saggi più importanti su ogni autore, e verso chi aveva messo a confronto la vita e le opere di artisti, scrittori e musicisti), ma soprattutto appare almeno dicutibile ritenere le riflessioni saggistiche di Rovani al di sotto dello spessore etico di Manzoni. Semplicemente, la lettura di Rovani della società italiana contemporanea parte da presupposti diversi e si propone mete etiche diametralmente opposte. Se sono innegabili la statura etico-civile e la complessità della visione del mondo di Manzoni, ci sembrano altrettanto innegabili lo spessore intellettuale di Rovani e l'articolazione davvero nuova della sua proposta.

Ma la bocciatura più netta è quando Baldi (1967: 101) sostiene che i Cento anni certamente non è un romanzo secondo quanto la critica si aspettava da un romanzo, ma non è neanche «più che un romanzo» come Rovani si era proposto. Ed è per questo che Baldi giunge alla sua interpretazione critica così felice nella storia della lettura dei Cento anni: essi non sarebbero un romanzo, bensì l'ipotesi di un romanzo, cioè, il terreno saggistico-narrativo nel quale Rovani esplora le possibilità di un romanzo, ma che egli stesso non avrebbe saputo costruire (Baldi 1967: 65-103). Infine, il critico concede al testo di Rovani il merito di aver tentato l'impresa: «In questa temperie di gusto, il tentativo generoso, anche se sfortunato, di Rovani, può sollecitare un nuovo interesse, e deve essere posto, accanto al capolavoro manzoniano, nella luce che gli spetta» (Baldi 1967: 103).

Da tutta un'altra angolazione, quella che vuole finalmente dispiegare le ragioni storico-culturali nonché estetiche dell'appartenenza o meno di Rovani alla Scapigliatura, Mariani, documenti alla mano, setacciata ogni posizione critica degli Scapigliati stessi, introiettata la prima critica di Nardi (Nardi 1923 [1968]) sulla Scapigliatura, considera Rovani, così come lo avevano considerato essi stessi, il capostipite della scuola. Ma a servire da pezza d'appoggio è, in fondo, l'affermazione di Albertazzi, ne Il romanzo (1903), secondo cui «della bohème milanese la maggior opera furono $\mathrm{i}$ Cento anni» (Mariani 1967: 55). E tale posizionamento colloca l'autore milanese come modello di chi ha costruito personaggi d'indole e carattere immorali o amorali e di chi ha avuto il coraggio di descrivere una società in disfacimento.

Per Mariani, Rovani è debitore di Balzac, nella misura in cui si propone di tracciare un itinerario narrativo basato sull'osservazione della società nell'immediato passato e nel presente, ma senza riuscirci del tutto, in particolare, per il disinteresse di Rovani verso la classe borghese. Anche questa affermazione è discutibile, posto quanto Rovani abbia insistito nel cambiamento di paradigma storico-culturale, che nel giro di pochi anni era passato dalla società aristocratica foscoliana, fondata sulla convinzione "italo-greca" della penisola, alla nuova società borghese dipendente dalle discussioni e dai dibattiti francesi (Rovani 1874a,1874b). Va ricordato che anche per Baldi è proprio l'interesse per la classe borghese a fare la differenza tra Rovani e il resto degli autori suoi contemporanei. D'altra parte, sempre secondo Mariani, Rovani sarebbe debitore di Stendhal, nello studio della potenza vitale di un individuo e nello studio delle sue passioni. Il distacco rovaniano da Balzac, infine, sarebbe dettato dal primato della storia come lettura del mondo nell'italiano, anziché dall'approccio antropologico e sociologico nella letteratura del francese. Quello che accomunerebbe entrambi è la convinzione che la penetrazione psicologica dei perso- 
naggi è una chiave di accesso allo studio del rapporto tra individuo e società. Certo, secondo Mariani, a Rovani rimane impressa piuttosto la lezione manzoniana, che non si discosta mai da una vocazione storiografica sovrapposta a quella narrativa, mentre in Balzac è la vocazione narrativa a imporsi su quella storiografica.

Fatto sta che i Cento anni sono stati visti da Mariani come un romanzo poco riuscito, nel quale si mescolano sia il resoconto cronachistico sia la narrazione fantastica, e nel quale il disegno della storia di Milano appare scialbo o addirittura confuso. Come si vede, la posizione di Mariani non è poi così distante da quella di Baldi. Si tratta sempre di un romanzo cui manca qualcosa o il cui unico pregio è di aver tentato un'impresa irraggiungibile.

Non solo. Per Mariani (1967: 140-142), se l'obiettivo ultimo dei Cento anni era restaurare il vero che la storia ufficiale non racconta, alla fine il vero non sarebbe altro che un insieme di vedute ideologiche dell'autore prestate ai suoi personaggi. Il testo di Mariani è disseminato di termini e di sintagmi che conducono ad una visione negativa del romanzo, che qui mi limito ad elencare: «ipoteca», «tentativo», «mancanza di armonia», «confusioni di stile», «tendenza alla digressione», «sottofondo pedantesco», «prova di equilibrio», «faticosa e pesante orditura», «pagina prolissa», «mescolanza», «insufficiente respiro narrativo», «fragili definizioni delle ragioni teoriche», «sacrificio delle vicende private», «astratto saggista», «selva del racconto», «impianto frammentario», «assenza di racconto», «groviglio dei fili», «scena affollata», «ammucchiamento di personaggi su personaggi», «surrogato dell'inesistente narrazione», «periodi mastodontici», «pura divagazione», «gusto della storia-pettegolezzo», «artificio provvisorio», «figura in bilico», «interminabile sacca» (Mariani 1967: 126-166). La sua metodologia, come si evince da questo campo semantico, ripete uno dei tic della tradizione critica nei confronti di Rovani: sottometterlo alla prova di confronto con Manzoni, come nel caso di Croce, Mazzoni, Borlenghi e, suo malgrado, Baldi. Merito però di Mariani è l'aver finalmente focalizzato gli aspetti squisitamente narrativi del romanzo.

Poi, mentre Baldi critica alla fine l'impianto romanzesco, salvando invece tutti gli interventi metaletterari come tracce di un vero laboratorio compositivo, Mariani annovera anche gli interventi di riflessione letteraria tra le pagine inutili e farraginose dell'insieme: le «dichiarazioni teoriche hanno invece la funzione di gabellare per innovazioni rivoluzionarie quelle che in realtà sono soltanto pretese soluzioni originali che sulla pagina si risolvono in fuoco di paglia, in artificiosi passaggi - che lo scrittore considera ardue dissolvenze tecniche - da un piano all'altro del racconto» (Mariani 1967: 164-165).

Secondo Folco Portinari (1970: 13), Rovani si trova in un clima culturale di sconquassamento della «struttura classica e tradizionale del romanzo». Dunque, è il primo a riconoscere che la grande sfida sia consistita nel superare una forma-romanzo resa ormai irripetibile dalla tradizione stantia e iterativa degli anni '30 e ' 40 . Il suo giudizio, esclusivo sull'opera narrativa, finisce per ammettere che i Cento anni altro non sono che «una sorta di inconsueto romanzo-saggio di inconsuete dimensioni» (Portinari 1970: 13), dove la parola inconsueto, nell'universo critico di Portinari, non necessariamente ha un saldo negativo. Anzi, il critico aggiunge:

Il bello e il nuovo dovrebbe stare nel gioco rovaniano di costruire, veramente, una tensione, comica o drammatica, per romperle subito e di continuo, così come di continuo è rotto il tessuto narrativo, la trama, in virtù di una disinvoltura, magari 
appresa dai modelli maggiori d'Oltralpe (da Sterne a Richter a Dickens...), ma impiegata con prodigalità anche eccessiva, in un perdersi e ritrovarsi tra un pettegolezzo gustoso, un saggio edudito, un aneddotto brillante (Portinari 1970: 13).

Insomma, Portinari segue la strada aperta da Baldi e Mariani, che hanno tentato di leggere Rovani en arrière, cioè, alla luce di ciò che era successo immediatamente dopo il discorso sul romanzo storico manzoniano e prima della composizione dei Cento anni, o en avant, cioè, dalla prospettiva scapigliata degli anni a venire, intendendo il capolavoro rovaniano non come l'impresa impossibile della rielaborazione tortuosa dei Promessi Sposi, bensì come "oggetto nuovo", capace di mettere in discussione il già acquisito e in grado di generare nuove ricerche.

Sarà Silvana Tamiozzo Goldmann a provare definitivamente per la prima volta una disamina del «romanzo totale» nelle sua logica compositiva e non a partire dagli schemi degli altri o della tradizione consolidata: «i problemi che Rovani pone allo studioso sono affrontabili pressoché solo attraverso la lettura delle sue opere» (Tamiozzo Goldmann 1994: 23). Il monito non è banale nel caso di Rovani, i cui critici hanno voluto attingere di qua e di là anziché al sistema coeso e coerente che egli stesso aveva creato. In questa direzione, si collocano molti dei giudizi di Tamiozzo (1994: 26): il ruolo assegnato a Rovani come capostipite della scapigliatura come «necessità di sostituire il mito manzoniano»; l'assoluta filiazione tra le tesi di Cattaneo e quelle di Rovani, nonostante la mancanza di documentazione che attesti i loro proficui incontri; la rilettura del legame con Manzoni, cui Rovani «contraddiceva proprio sul versante esistenziale, della vita pratica, sottraendosi - non solo per disparità artistica - ad ogni possibilità di assimilazione con 1'autore dei Promessi Sposi e con la sua visione del mondo» (Tamiozzo Goldmann 1994: 27); e, infine, la considerazione dei Cento anni come «una struttura aperta e duttile, decisamente originale rispetto alle esperienze coeve e alle sue stesse precedenti» (Tamiozzo Goldmann 1994: 35). Tale giudizio nettamente positivo è seguito da una puntuale ricognizione analitica delle strategie e delle soluzioni narratologiche, delle tematiche affrontate, dell'articolata funzionalità dei personaggi, elementi tutti che fanno dei Cento anni un romanzo davvero straordinario. La posizione di Tamiozzo viene ribadita nell'ultimo saggio su Rovani (2018) all'interno del recente volume sull'Ottocento italiano curato da Alfano e De Cristofaro (2018).

Nella sua fittissima storia del romanzo, Tellini (1998: 106-112) torna alla comparazione tra i Cento anni e Le confessioni d'un italiano e, pur sottolineando quanto i due romanzi siano strategicamente diversi, smonta alcuni punti chiave della critica rovaniana: il filone etico-civile e l'attacco frontale all'architettura del romanzo. Infine, anche se Tellini (1998: 110) riconosce che i Cento anni «non sono un esperimento mal riuscito bensì un'operazione indisciplinata e magmatica», il romanzo non rappresenta a suo avviso niente di nuovo, ma la dissoluzione del vecchio. Il suo merito sta nel fatto che «lo sparpagliamento dell'edificio tradizionale e dei suoi arredi, lascia presagire che il nuovo è alle porte». Ma il nuovo non è Rovani.

Per finire, Folco Portinari (2008: xv), nell'ultima edizione italiana dei Cento anni, torna sulle parole di Baldi, il cui volume è, a suo avviso, «ancor oggi il più penetrante e convincente sul Nostro», ricordando però che Angelo Romanò (1958), nel suo studio sulla narrativa del secondo romanticismo lombardo, aveva assolto lo scrittore milanese da tante accuse rivoltegli e aveva dimostrato quanto il clima culturale cattolico della Milano di quegli anni non gli fosse stato favorevole (Romanò 1958: 26). 


\section{Storia e statuto del romanzo}

È il caso ora di soffermarsi sul ruolo che Le tre arti, il compendio di teoria e di critica letteraria, musicale ed artistica, ha avuto nell'itinerario culturale di Rovani. Basti ricordare qui che molti dei saggi contenuti ne Le Tre Arti, come abbiamo detto, erano già stati pubblicati nell'ultimo volume della Storia delle Lettere e delle Arti, ma soprattutto che la sua ideologia estetica fosse il risultato di anni di critica militante nei giornali lombardi, attività frenetica che Valentino Scrima (2004) ha riscostruito nel suo volume sulla critica rovaniana.

Lo studio delle arti è caratterizzato da una prospettiva storica, che consente a Rovani di inanellare capitolo dopo capitolo il loro sviluppo espressivo. Chiarito lo schema evolutivo entro cui ogni opera si colloca, scopo fondamentale del testo è riflettere sullo statuto intrinseco ad ogni «forma espressiva» (romanzo, poesia lirica, ballata, satira, drammaturgia, ma anche pittura, scultura, opera lirica, sinfonia ecc.). Partendo dalla conferma, rigorosamente documentata, che a partire dagli anni '50, Rovani diventa «il critico più temuto d'arte in città», Scrima (2004: 8) sostiene che

Rovani sorprende i lettori dell'epoca con la vivacità del suo estro. Inaugura un modo di fare giornalismo più spigliato e cattivante, lasciandosi alle spalle il modo saggistico austero e referenziale di Defendente Sacchi, Pietro Selvatico e Carlo Tenca, per spianare la via ad una critica nuova, più battagliera, a volte intenzionalmente controcorrente.

Per Scrima, in poche parole, affrontare i testi rovaniani senza conoscere la sua prosa giornalistica da critico militante (come la chiameremmo oggi) «è quantomeno rischioso» (Scrima 2004: 13). A tale premessa, ci si consenta di aggiungere che giudicare la narrativa rovaniana senza considerare la sua posizione teorica sul romanzo (e più estensivamente sulla letteratura e sull'arte), elaborata scrupolosamente negli anni tra i primi tre romanzi storici e i Cento anni, sia negli scritti giornalistici non inclusi ne Le Tre Arti e che Scrima recupera, sia nei saggi che convergono nel volume postumo, è, oltre che rischioso, fuorviante.

Non è una novità della critica l'ammirazione di Rovani per la prosa e per le idee di Cattaneo, sin dagli anni della reciproca frequentazione nell'esilio svizzero di entrambi. Non sono neanche nuovi ormai gli accenni a come la collaborazione di Rovani presso lo Spettatore industriale, periodico nell'orbita culturale del Politecnico di Cattaneo, sia stata determinante per suscitare nell'autore dei Cento anni l'interesse per l'architettura e l'urbanistica, ma soprattutto l'interesse per i modi in cui Milano, trasformandosi, non dovesse rinunciare alla propria tradizione (Scrima 2004: 17-18), al punto che lo stesso concetto di storia sarebbe di derivazione cattaneana: «Il presente di Milano deve radicarsi pienamente sulla tradizione, se vuole incamminarsi senza danni sull'erta dell'incivilimento» (Scrima 2004: 19). Così come sono cattaneani la destinazione sociale dell'arte, l'impegno civile dell'intellettuale, la fine formativa delle scienze umane, lo sviluppo artistico come progresso della Nazione.

La precisazione più importante della solidisssima ricostruzione storico-artistica di Scrima è la collocazione dell'influenza di Rovani in un solo decennio, gli anni '50, dopodiché la sua visione dell'arte, così come la sua capacità di interferenza nell'am- 
bito estetico lasciano spazio ai nuovi critici degli anni '60 in poi: «[Rovani] ambisce alla spregiudicata carica di estetologo ufficiale della Milano radicale tra gli anni Cinquanta e Sessanta» (Scrima 2004: 30).

Quello che è stato osservato solo da Scrima - e, a nostro avviso, giustamente - è che Le Tre Arti è, innanzitutto, anche se molte volte tra le righe, un vero trattato di poetica, oltre che un «discorso critico organico» (Scrima 2004: 74), in cui si definiscono uno ad uno, come abbiamo detto, i diversi generi, dalla lirica alla satira, dal romanzo al sermone, dall'opera lirica alla sinfonia, dalla scultura alla pittura.

Se per Baldi i Cento anni non sono altro che l'ipotesi di un romanzo, per noi è piuttosto nelle pagine teorico-critiche de Le Tre Arti che Rovani affronta la storia e lo statuto del romanzo e, quindi, esse sono lo spazio saggistico in cui egli dà luogo ad una riflessione metaletteraria, di cui i Cento anni sono in parte il risultato. Quindi, semmai, per rovesciare le parole di Baldi, a nostro avviso, Le Tre Arti è l'ipotesi e i Cento anni la tesi rovaniana: un romanzo frutto delle riflessioni avanzate nel suo studio complessivo sull'arte. Non si può essere del tutto d'accordo, quindi, con Tamiozzo Goldmann (1994: 35) quando afferma che «Rovani è tuttavia restio a dichiarare la propria metodologia di lavoro» né quando, riferendosi a Le Tre Arti, asserisce che si tratta di un percorso che non illumina la sua costruzione narrativa. Per noi, invece, sono proprio le riflessioni di Rovani attorno allo statuto del romanzo - oltre che di altre «forme espressive» - ad illuminare la sua stessa impresa narrativa, sia quella precedente, sia quella in atto, sia quella a venire ${ }^{4}$.

Per meglio capire quali siano i nodi della sua trattazione teorica sul romanzo, ci si propone di dividere l'analisi in tre punti: metodo d'indagine, canone italiano, visioni e concetti.

\subsection{Metodo d'indagine}

Le pagine introduttive della Storia delle Lettere, riprodotte ne Le Tre Arti, partono dal presupposto che le arti - «la parola, la plastica e la tonica» (Rovani 1874: v) avanzano simultaneamente in un percorso di incivilimento delle nazioni. A forgiare la letteratura convergono la storia e la filosofia, fonti alle quali essa s'abbevera: la sua capacità di diffusione sarebbe più scarsa se non intervenisse la musica, che rende accessibile ad un pubblico più vasto ed universale le sue ricerche e le sue scoperte. Ora, allo scopo di penetrare nella mente e nell'animo del pubblico, l'inafferrabilità fisica della musica ha bisogno della corporeità di altri linguaggi artistici, quali la pittura e la scultura, affinché la parola trovi nuovi canali più stabili e duraturi.

Due osservazioni ci interessano. La prima è che esiste un filo rosso inscindibile (storia-filosofia-lettere-musica-arte), che conduce implicitamente ad un ragionamento teorico poche volte esplorato nelle analisi delle sue opere: non la corrispondenza delle arti in chiave simbolista, ma la convergenza dei discorsi - diremmo oggi - storici, filosofici, letterari, musicali e plastici nei suoi romanzi. Per esempio, i Cento anni non sono mai stati studiati in quanto romanzo illustrato, nonostante le ottantaquattro incisioni originali, corredate di altrettante immagini secondarie che arricchi-

4 In un altro saggio, attualmente in costruzione, mi propongo di analizzare i primi tre romanzi come laboratorio d'idee estetiche che convergeranno nell'opera maggiore. 
scono il testo ${ }^{5}$. Così come non si è mai fatto accenno alle innumerevoli annotazioni musicali che dovrebbero, secondo la sua strategia narrativa, accompagnare alcuni frammenti della sua opera. La seconda osservazione è che, secondo Rovani, solo l'Italia vanta uno sviluppo artistico in cui le arti hanno subito un processo evolutivo nel quale storia, filosofia, lettere, musica e arti figurative si sono alimentate in modo prodigioso nel tempo, a tal punto che una considerazione critica presumibilmente relativa ad una delle espressioni estetiche sotto esame non può prescindere mai da ciò che una delle «arti sorelle» ha manifestato contemporaneamente. Sappiamo che il punto culminante di quest'ottica sarà la costruzione di alcuni binomi, che a detta di Rovani, sostengono l'edificio ottocentesco (Manzoni-Rossini, Grossi-Bellini, Vela-Verdi). Ma non sono tali binomi ciò che a noi interessa, bensì le strategie che approdano ad essi e, per la precisione, alcuni punti chiave della sua trattazione, ai fini di analizzare puntualmente cosa fosse o cosa dovesse essere negli anni ' 50 , in seguito al monito manzoniano, il romanzo.

Perno centrale della trattazione teorica è la biografia, intesa, in senso ottocentesco, come discorso organico attorno al profilo intellettuale di un autore e all'analisi delle sue opere. La biografia rovaniana poi ha una scansione quasi regolare, composta di paragrafi che, anche se contigui, rispettano un'organizzazione logico-consequenziale: collocazione di un autore in un luogo e in un determinato tempo; indicazione della filiazione poetica dell'autore, della sua incidenza sui dibattiti attivi o passati e della loro capacità di dialogare con la tradizione; analisi delle opere in funzione di ciò che hanno apportato di nuovo al sistema; reazioni del pubblico; infine, ruolo dell' autore all'interno del canone. Come egli stesso fa capire, fonte della sua attività d'indagine critica rimane il capolavoro di Antonio Zoncada, I Fasti nelle Lettere in Italia nel Corrente Secolo (1851), il cui merito era quello di presentare le biografie degli scrittori «in modo che gli studiosi potessero farsi un'idea di ciascun scrittore, ma i loro frammenti uniti, servissero a presentare quasi un secolo completo sia di storia, che di letteratura, che di critica e di poesia» (Rovani 1874a: 217).

Presupposti di questo metodo sono la compattezza e il predominio del mondo milanese dell'Ottocento (anche in campo pittorico), la già segnalata dialogicità tra le arti, la supremazia della parola sulle altre forme. Un tale impianto consente a Rovani di costruire un vero universo culturale tra gli anni ' 20 e gli anni' 60 dell'Ottocento, che risulta forse una delle letture più stimolanti (e anche polemiche) sulla contemporaneità ad opera di un autore allora vivo.

\subsection{Canone ottocentesco}

Nelle ultime pagine della prima parte de Le Tre Arti, dedicata alla letteratura, e nelle prime pagine sia della seconda parte che della terza parte, dedicate rispettivamente alla musica e alle arti figurative, Rovani insisterà sempre di più sull'idea di un predominio, anziché primato, delle teorizzazioni e delle esplorazioni estetiche in ambito lombardo, di cui Roma, Firenze, Napoli e Venezia saranno satelliti. Perché al di là

\footnotetext{
Mi sono proposto in un altro saggio, in via di pubblicazione, di affrontare per la prima volta il rapporto tra testo alfabetico e testo visivo nell'edizione definitiva dei Cento anni, pubblicata in vita dell'autore presso Redaelli nel 1868-1869.
} 
dell'origine geografica degli artisti, sarà Milano a conferire quella legittimità critica che equivale a ciò che Parigi significa per la consacrazione europea.

Per poter affermare tale posizione Rovani ricorre dunque ad una stratificazione cronologica delle opere, che dimostri efficacemente quanto la Lombardia, dotata di caratteristiche specifiche, finisca per modellare un determinato "pensiero" estetico tradotto poi in arte. Affinché ci sia un decorso evolutivo ci deve essere una genesi, un punto di partenza, uno sviluppo e, magari, un punto di arrivo. E, quindi, ci deve essere una rivoluzione:

La rivoluzione della letteratura italiana era già del resto incominciata assai tempo anzi la venuta di questi forti ingegni [Monti, Foscolo], e, fin da quando una giovane schiera di pensatori erasi raccolta a Milano a discutere in casa Verri, lo studio delle cose utili aveva già iniziata quella letteratura severa, in cui Parini aveva fatto le prime e potenti sue prove. Quei critici che, non so per che fini o per qual capriccio, si tormentano nel voler che l'Italia, dopo il secolo decimoquinto, siasi fatta in ogni cosa imitatrice delle altre nazioni, sono quei medesimi che vorrebbero far derivare la nuova scuola capitanata da Manzoni dalla cognizione profonda delle letterature straniere, la francese specialmente e la germanica, e non già dai primi lampi innovatori che uscivano da quelle opere dove è più profondo il suggello dell'ingegno di Monti e Foscolo (Rovani 1874a: 4).

Questo esordio pone, come si vede, due problemi: la discussione con la critica attorno all'italianità insita nella rivoluzione letteraria in atto nei primi anni dell'Ottocento e la genealogia costitutiva del sistema. Il primo punto riguarda innanzitutto il ruolo che assume Foscolo. Bisogna ricordare che nei Cento anni Rovani non solo menziona Foscolo come autore, ma lo rende personaggio vivo della storia. Su Foscolo pesa il giudizio negativo su Milano («la Babylo minima»), giudizio che lo stesso romanzo di Rovani cercherà di correggere, mettendo Milano al centro indiscutibile della genesi e dello sviluppo della modernità. Poi, mentre Manzoni, D’Azeglio, Grossi, Pozzone (il suo noto maestro al liceo), Giunio Bazzoni, Cantù, Prati, Aleardi, Carrer, Leopardi, Giusti, Revere, Poerio, Torti, Uberti, Zoncada, Gian Battista Bazzoni, Zanoia, Guerrazzi, Porta, Riaberti hanno avuto un saggio specifico ne Le Tre Arti, Foscolo è affrontato indirettamente a partire dall'irruzione di Manzoni sulla scena italiana ${ }^{6}$. Foscolo è il sintomo del nuovo, Manzoni la genesi.

Tale canone critico, secondo una prospettiva storica che tiene conto in genere del succedersi delle opere più che degli autori, non condivide il giudizio diffuso secondo cui la letteratura italiana dell'Ottocento, al solo scopo di innovare, abbia dovuto attingere a fonti straniere. Per Rovani, come si evince dal frammento citato, la fase di rivoluzione, trasformazione ed evoluzione del sistema letterario moderno è avvenuta secondo un processo di assorbimento delle forze interne alla letteratura italiana, e solo in seconda istanza delle forze straniere. Il dibattito aperto è con la generazione romantica (i vari Berchet, Borsieri, di Breme, Pellico, Visconti, Confalonieri), vistosamente assenti dai suoi Cento anni (a eccezione di Confalonieri, ritrattato come un padre e marito perverso) e da Le Tre Arti. La ragione dell'e-

6 Naturalmente, nella Storia delle lettere, suo compendio monumentale sulle biografie dei più grandi intellettuali italiani nel tempo, Foscolo è messo all'inizio del IV volume, ma affrontato da un saggio critico altrui, senza indicazione di fonte. 
sclusione è chiara e dimostra la presa di posizione di Rovani nei confronti dell'ormai vecchio dibattito aperto da Madame de Staël attorno all'importazione di altri modelli culturali. Abbiamo detto che nelle analisi delle opere, Rovani presta attenzione sia all'evoluzione interna di un autore ma anche all'evoluzione esterna del sistema cui quell'autore appartiene: quindi, alla collocazione precisa di un testo entro un determinato periodo compositivo, lo scrittore milanese aggiunge la filiazione letteraria dell'autore, con un'attenzione quasi ossessiva nell'individuare ogni volta quali siano le fonti che provengono direttamente dalla tradizione italiana, anziché dalla tradizione francese, inglese o tedesca. Ciò non significa che Rovani si collochi in una posizione ingenuamente nazionalistica: è dalla sua frequentazione della cultura tedesca e francese che nascono le osservazioni più interessanti sull'identità del sistema culturale italiano. Per esempio, contro la tendenza della letteratura italiana dell'Ottocento alla critica sociale in chiave francese, lui rimanda alla lezione pariniana, dal momento che, alla lettura dei classici come fonte di ispirazione permanente, sarebbe subentrata la «polvere enciclopedica» (Rovani 1874a: 25), intesa da Rovani come accumulazione nozionistica senza gerarchia e, soprattutto, senza stile. Atteggiamento enciclopedistico che, paradossalmente, molta critica gli ha attribuito.

La costruzione del canone avviene secondo gli equilibri tra geni e ingegni. La teoria del genio ha la sua genesi moderna nelle lunghe discussioni del gruppo dell'Encyclopedie, che alla fine convergono nella nota definizione filosofica, oltre che storica e mitologica, del génie:

L'homme de génie est celui dont l'ame plus étendue frappée par les sensations de tous les êtres, intéressée à tout ce qui est dans la nature, ne reçoit pas une idée qu'elle n'éveille un sentiment, tout l'anime \& tout s'y conserve. Lorsque l'ame a été affectée par l'objet même, elle l'est encore par le souvenir; mais dans l'homme de génie, l'imagination va plus loin; il se rappelle des idées avec un sentiment plus vif qu'il ne les a reçûes, parce qu'à ces idées mille autres se lient, plus propres à faire naître le sentiment (Encyclopedie 1757: 581).

Contro la visione enciclopedistica, Rovani (1874b: 9) afferma che il genio è colui che «è essenzialmente invasore e conquistatore. Egli fa suo tutto quello che trova per via» al contrario degli ingegni che «si lasciano trasportare dal suo carro falcato», perché la natura «non diede loro tal forza da fermare l'altrui velocità». Il genio non è solo quello che fagocita tutto ciò che è tradizione per creare di nuovo qualcosa di unico, è quello la cui sensibilità «s'inflette a tutto e assume tutte le movenze del pensiero» (Rovani 1874b: 11). Quindi, più che una periodizzazione per decenni, per epoche o per scuole, Rovani divide il percorso della cultura ottocentesca secondo la dominazione in ogni genere da parte di un genio e in funzione dell'affastellamento di forti ingegni, involontariamente incapaci di svoltare pagina. Ancora più chiaro il suo intervento sul genio nel capitolo su Rossini :

Il genio riguarda l'idea madre, il complesso architettonico di un'opera. Esso sorveglia al tutto, mentre l'estro non si occupa che delle parti. Il genio è creatore, è originale, è caratteristico [nel senso che oggi daremmo alla parola identitario]; invade, domina e non piega innanzi a nessuna autorità. [...] Murat non è Bonaparte (Rovani 1874b: 14). 
Ma la particolarità del genio non è insita solo nella sua battaglia avanguardistica, bensì nella sua capacità di rinnovarsi esso stesso, mettendo in dubbio i suoi stessi passi: «Il genio continua a fare per far meglio, o per far diversamente. Perché si tedia e arrosisce di ripetere la stessa cosa e non poter battere una via nuova» (Rovani 1874b: 27). E, per finire, «un altro sintomo che accusa la presenza del genio è quello di saper ritirarsi a tempo» (Rovani 1874b: 29). L'ingegno, invece, è il complemento necessario del genio in ogni sistema culturale. Ad esso tocca offrire una parte complementare del disegno ambizioso di un'opera che aspira alla totalità, parte che non viene dissacrata da Rovani, ma considerata elemento essenziale al tutto. E se un'altra caratteristica del genio è saper esercitare il riso e il pianto, «gli ingegni non sanno fare altro che ridere o piangere» (Rovani 1874b: 32). Per tutto ciò, la definizione degli ingegni artistici finisce per chiarire l'estetica rovaniana: essi «sono destinati ad essere ingegni di memoria, di dottrina e di combinazioni, oratori felici di ciò che fu trovato da altri» (Rovani 1874b: 37).

Modello geniale del processo costitutivo della nuova letteratura è Manzoni, il quale, anziché aver imitato o riformulato le soluzioni che gli venivano offerte dai prodotti stranieri (i noti Scott, Goethe e Chateaubriand, nella visione di Rovani), portò a compimento un'operazione vastissima di rinnovamento in tutti gli ambiti della letteratura che né la lezione pariniana né l'impeto alfieriano né il tentativo foscoliano erano riusciti a portare avanti. Si tratta, quindi, di confermare il carattere fondativo della modernità manzoniana sia nel teatro, nella lirica, nella filosofia della storia e, chiaramente, nella prosa, capace ormai di percorrere «tutti i toni e [di] riflettere l'indefinita varietà della vita» (Rovani 1874a: 8). Tutto ciò fu possibile perché, all'indomani dei tre grandi autori prima menzionati, Manzoni colse «il segreto dell'esistenza della sua lingua, di cui per tanti secoli si andò affannosamente in cerca, senza sapere mai dov'ella fosse» (Rovani 1874a: 8).

Ma al di là di queste constatazioni, tra l'altro riconfermate dalla critica sia a lui coeva che futura, l'originalità dello sguardo di Rovani sta nell'affidare a Manzoni uno sguardo severo che non si compiange nell' evocare una grandezza passata, bensì nel mettere a nudo le fonti e l'essenza dei mali che affliggono la nazione. Rovani chiama questa capacità introspettiva della letteratura manzoniana la «coscienza della storia» (Rovani 1874a: 8), che invece nega al pur stimatissimo Leopardi.

Più che considerare in questo panegirico - come lo ha considerato la critica - solo una presa di posizione a favore dell'opera di Manzoni contro i suoi detrattori, a cominciare da Foscolo, sarebbe il caso di vedere come in esso Rovani fondi le sue convinzioni teoriche sulla letteratura:

Un libro unico come i Promessi Sposi non solo è destinato a rimanere unica produzione della mente di un uomo, ma unica produzione eziandio di un intero periodo letterario, e le ragioni di questa unicità stanno appunto nell'originalità sua, nella novità dell'assunto, nell'universalità degli elementi, nello specchio sincero e amplissimo della vita e degli uomini (Rovani 1874a: 25).

Ciò che bisogna chiarire quindi è in che cosa consista «l'originalità sua», al di là di ciò che lo stesso Rovani ha già espresso. Essa è implicita nel suo saggio in vari punti. In primo luogo, i Promessi Sposi sono «il risultato finale di tutta la sua esperienza e di tutte le sue meditazioni» (Rovani 1874a: 25). L'affermazione potrebbe sembrare scontata, ma provenendo da Rovani, va letta nell'ottica di chi ha assegnato 
al vissuto degli scrittori, non un mero carattere di testimonianza intellettuale, bensì un valore inestimabile e incontrovertibile di "esperienza" capace di tradursi in arte. Un punto essenziale della poetica rovaniana è, precisamente, l'articolazione tra narrazione e biografia. In secondo luogo, la poetica manzoniana non va giudicata sulla base dell'esito raggiunto da ogni singola opera, ma nel suo insieme e, soprattutto, come un'architettura coerente e coesa, in cui ogni pezzo compromette l'intero edificio. Per esempio, facendo la rassegna delle opere in evoluzione, Rovani constata che in Manzoni c'è un'indagine storica capace di «riempire tutte le lacune della storia» (Rovani 1874a: 27). In terzo luogo, la Storia della colonna infame sarebbe - e in questo Rovani ha anticipato la critica contemporanea che ormai ha assunto questa posizione - non un excursus, ma una riflessione conclusiva del romanzo, che rifiuta qualsiasi giustificazione compiacevole di un esercizio della giustizia che non si attenga scrupolosamente alla legge. E, infine, il Discorso sul romanzo storico non sarebbe, come molti contemporanei lo giudicarono, un'autocritica manzoniana del suo capolavoro o una rinuncia inesorabile a un determinato esercizio narrativo in chiave storica, bensì una riaffermazione da parte del venerato scrittore milanese del rigore storico su cui deve basarsi anche un romanzo, al di là dell'orizzonte narrativo dettato dalla fantasia. A tal punto che:

Che cosa fa la storia, anzi la filosofia della storia? Discute sul modo di unire gli sparsi anelli e di completarli in serie, e conchiude con qualche opinione: lo storico, sia il più sagace, non fa nulla di più; fa di un periodo quel che farebbe un archeologo del torso del Belvedere, non eseguendone il ristauro, ma proponendo congetture e ipotesi sulla struttura e condizione della statua intera. Ma l'artista va più in là dell'erudito, compiendo con un proposito unico e determinato quel che l'altro appena accennava dubitante fra molti propositi. Né ci pare che l'artista andando più in là dell'erudito, per troppa audacia corra pericolo d'ingannare l'osservatore (Rovani 1874a: 38).

Se ora noi volgiamo tutte queste interpretazioni verso il laboratorio rovaniano emergono alcuni punti saldi della sua poetica. Primo, i Cento anni sono, come i Promessi Sposi, il risultato finale della sua esperienza di vita e di studioso. E tali strade non vanno disgiunte, ma non vanno neanche celebrate una distante dall'altra, come alla fine fece, pur senza calcolare l'esito delle sue passioni, lo stesso Dossi, il quale, come si sa, finì per oscurare l'opera in funzione del personaggio. Secondo, assunto il romanzo come il suo capolavoro indiscusso, non bisogna mai perdere di vista che lo scrittore ci arrivò dopo un lungo percorso esplorativo, in cui ogni parte ha una sua funzione. Nel caso della narrativa storica, pertanto, a noi non sembra, come si evince ormai da un luogo comune della critica, che i Cento anni siano un superamento e archiviazione dei tre romanzi precedenti, ma semmai il completamento di un itinerario coerente e coeso. Perché se Manzoni coprì, secondo Rovani, tutto il passato d'Italia dall'Adelchi ai Promessi Sposi, lo stesso Rovani disegnò un proprio itinerario storico che parte dalla Valenzia Candiano per approdare al mondo della massoneria ne La Libia d'oro e, infine, per affrontare, in chiave volutamente moderna, Giulio Cesare - a detta di Rovani il maggior personaggio dell'antichità - còlto però nel pieno della sua gioventù. Quindi, non un romanzo finale, costruito sull'affabulazione stanca della Roma antica, ma l'ultimo stadio della sua poetica, che rimandava a questo punto all'origine di tutto. 
A ben guardare, tutto questo percorso è in se stesso un altro tentativo di colmare le lacune lasciate da Manzoni. Rovani, una volta giunto alla contemporaneità, in perfetta coerenza, torna indietro e chiude il proprio itinerario con la giovinezza di Giulio Cesare, vero approdo finale di uno sguardo che più che storico è diventato addirittura archeologico, in quanto direttamente collegato alla fonte della storia d'Italia. Nelle seguenti parole di Rovani (1874a: 41) attorno al Dialogo dell'Invenzione di Manzoni, «non è ridicolo occuparsi anche del primo anello della catena a cui è attaccata l'àncora del galleggiante naviglio», per noi è chiara l'esplicitazione finale di un metodo. Certo, Manzoni si riferiva al fatto che la filosofia italiana moderna, accusata di non essere stata alla pari di quella francese, forse ha fatto bene a non dipendere da un sistema di pensiero che, secondo lui, da Rousseau giunge a Robespierre. Ma la lettura che ne fa Rovani è un'altra: la cultura italiana deve misurarsi con quanti dibattiti, quante idee, quante opere provengono dall'estero, per trovare, se non una sua soluzione, almeno una chiave interpretativa all'interno del proprio sistema culturale. E poi, tornare alle origini, dove sono depositati i germi costitutivi di tale sistema. Non solo, La Libia d'oro sta a Cento anni come la Storia della colonna infame ai Promessi Sposi. Cioè, non solo come propaggine narrativa di un universo compatto, bensì come suggello saggistico - al di là della corposità narrativa - in cui le ipotesi di carattere filosofico e storico dei Cento anni vengono confermate, ampliate e disseminate nel nuovo testo.

Sarebbe però fuorviante, a nostro avviso, non ravvisare tutto ciò che distanzia Rovani da Manzoni, non solo in termini teorici, ma nella loro produzione letteraria tout court; distanza che non va misurata qualitativamente secondo il paradigma manzoniano. Nonostante la profonda ammirazione per Manzoni, Rovani, dal punto di vista narratologico, interviene nei Cento anni agli antipodi del suo amato maestro. In primo luogo, assegna ai personaggi storici (si vedano i capitoli in cui compare Pietro Verri) il ruolo di servire da "sfondo", in modo che emergano i veri personaggi e non i personaggi veri. È come se rovesciasse l'economia della narrazione manzoniana. Lo spessore va verso i fittizi, gli altri non sono dissimili dal ritratto che Rovani aveva già scritto ne Le Tre Arti ${ }^{7}$.

In secondo luogo, sono completamente distanti sia il narratore che il lettore di entrambi i romanzi. In Rovani, l'idea di un narratore onnisciente, non senza ironia, muta in una voce a volte distratta, divergente, digressiva, umorale e polemica. Così, il lettore, sentito come "asino" e diffidato con tono sarcastico pungente, non è condotto per mano, ma persino maltrattato, deriso, abbandonato a se stesso in una sfida del narratore davvero violenta.

In quanto alle strategie narrative, anche il rapporto con le arti è diverso. La recente pubblicazione del saggio di Daniela Brogi (2018) sui Promessi Sposi come

\footnotetext{
«Fra i personaggi, che sono già molti e saranno numerosissimi di questa nostra storia, e che non tengono da noi altro incarico, pur nella loro importanza drammatica, che di costituire la moltitudine ed il fondo ai veri grandi uomini storici dei cento anni decorsi, facciamo ora, per la prima, avanzare la figura giovanile di Pietro Verri, come antiste a quella schiera gloriosa di uomini grandi appunto e d'uomini utili, i quali e a gruppi e sparsamente e ad uno ad uno vedremo sorgere, come alberi di alto fusto tra la fitta selva delle piante volgari. - Essendoci proposti di mostrare in azione il più di questi benemeriti, per cui Milano e la Lombardia, e, rispetto a certi elementi speciali della vita pubblica, l'Italia tutta e persino l'Europa si atteggiò a vita più razionale, vedrem frattanto il giovane Verri a contrassegnare il suo primo ingresso tra gli uomini, con uno spirito già vigile a combatter le male consuetudini, per cui il secolo non poteva più reggersi, e col coraggio ad affrontar tutti gli ostacoli che i pregiudizi della sua casa, del suo ceto, del suo tempo dovevano opporgli onde farlo stramazzare a' primi passi» (Rovani 2008: 158).
} 
un romanzo concepito «per gli occhi» sembra avvicinare in realtà Rovani al suo autore preferito. Perché, come abbiamo detto, anche i Cento anni sono stati un romanzo illustrato. Ma, in realtà, mentre in Manzoni tale strategia deriverebbe, come dimostra saldamente Brogi, da una necessità di costruire un romanzo visivo che attinga il più possibile alle forme storiche della percezione e della comprensione del mondo in chiave popolare, in Rovani c'è un salto teorico che fa sì che ogni immagine, sia nel testo alfabetico sia nel testo visivo, risponda alla necessità di portare a compimento la sua idea cardine della reciproca alimentazione delle arti. Se Baldi, poi, sostiene che la corrispondenza fra le arti è solo simultanea, accostamento di espressioni coeve nel tempo, per noi, invece, Rovani riflette nel suo romanzo sulla eventuale possibilità di adoperare strategie compositive che provengano da altre arti:

La letteratura sarebbe assai più feconda se avesse il comodissimo privilegio della musica, nella quale, allorchè un maestro si trova a contatto di una bella situazione drammatica, e si ricorda d'aver letto in qualche vecchio spartito un bel motivo che gli paia ben adatto alla situazione stessa, se lo appropria senza molti scrupoli e senza timore che gli si possan fare i conti addosso (Rovani 2008: 191).

Certo, non mancano le affinità manzoniane: il tema dell'educazione (si ricordi la tenebrosa storia di Federico Confalonieri, ancora più cupa di quella di Gertrude), la questione dell'amministrazione della giustizia (bellissime le scene di ascendenza verriano-manzoniana sulla tortura inflitta a Galantino), la rappresentazione storica attraverso i personaggi minori, la comparazione tra Cristoforo e donna Paola Pietra, l'impianto storiografico compromesso dal piano narrativo. Ma tali affinità vanno nella direzione che lo stesso Rovani enuncia come principio di poetica: ogni romanzo che speri di diventare "canonico" si colloca in un punto della tradizione, da cui raccoglie esperienze e insegnamenti, ma di cui rifiuta formule e idee.

Se effettivamente l'alleanza tra Manzoni e Rovani fosse stata equivalente a quella del maestro e dell'epigono, così come in genere ha sentenziato la critica, oppure in termini rovaniani, tra il genio e l'ingegno, non si spiega perché Le Tre Arti, collocando l'opera di Manzoni all'inizio del percorso culturale, prosegua per almeno cinquecento pagine nella disamina di quello che è successo dopo Manzoni, né si capisce perché, appurata 1'unicità dei Promessi Sposi, Rovani voglia ripeterne il modello.

Vediamo, dunque, come si articola il canone dopo l'irruzione manzoniana. D'Azeglio andò oltre Manzoni, senza superarlo, perché, fu il primo che, cogliendo una lezione indelebile dalla propria produzione pittorica, cioè, imponendo al paesaggio la presenza degli uomini, al di là di ogni vedutismo (Rovani 1874a: 61), coll'Ettore Fieramosca introdusse l'effetto pittorico nella letteratura, ossia «i contrasti di ombra e di luce, e le descrizioni colorite con pompa di tavolozza e con la predilezione di quei particolari che chi non è pittore trascura e disprezza» (Rovani 1874a: 63). Penso non sia necessario riflettere su quanto fosse essenziale per Rovani che un romanzo contenesse al suo interno una dimensione plastica, e su quanto egli stesso ci abbia tenuto lungo tutta la sua produzione narrativa. Sorprendentemente, il capolavoro di D'Azeglio non sarebbe l'Ettore Fieramosca, ma il Niccolò dei Lapi, per tre motivi ben precisi: un impeto nazionalistico più solido, una più rigorosa costruzione storica e una maggiore perizia tecnica (Rovani 1874a: 64). 
La trattazione critica attorno all'opera di Grossi, in particolar modo l'Ildegonda e I Lombardi alla Prima Crociata, avviene secondo un confronto con i pregi e i difetti rilevati dalla critica precedente. Ma il punto fondamentale è che il discorso polemico conduce Rovani a stabilire quali siano le tre ragioni che devono convergere in un'opera ai fini del suo successo, nel senso che Rovani dà a questo termine: collocarsi definitivamente nel filo rosso della tradizione. Tali ragioni sono quella filosofica, quella poetica e quella artistica. (Rovani 1874a: 75) La prima non va intesa come impianto etico-civile, bensì come riflessione profonda circa l'individuo e la società del proprio tempo (anche se declinato al passato); la seconda vuol dire che ogni opera è compromessa dal proprio genere e, quindi, basata sull'equilibro tra forma raggiunta e forma nuova; la terza, di stampo rovaniano, va intesa come capacità del romanzo di evocare altre forme espressive e addirittura di generarle, offrendo al lettore ulteriori possibilità d'indagine sul mondo.

Se la Margherita Pusterla del Cantù viene alla fine riscattata come opera tutto sommato riuscita, non però in grado di collocarsi sul filo rosso della tradizione-innovazione, la Storia dei Cento anni (1851), occupandosi proprio del periodo che poi interesserà Rovani nel suo romanzo, viene liquidata, insieme alla Storia degli Italia$n i$, come non «altro che zuppe fatte con brodo attinto alla gran caldaia della Storia Universale» (Rovani 1874a: 104-105). Al di là del giudizio concreto, a noi interessa sottolineare che per Rovani l'opera complessiva di un autore è valida ogni volta che esso affronta in ogni sua pubblicazione non la stessa "zuppa", ma una nuova problematica secondo una nuova ottica. Il giudizio si conclude in una formula, già accennata, secondo cui il Cantù può essere considerato un ingegno, ma certamente non un genio capace di rovesciare la trama della tradizione ${ }^{8}$. La stessa categoria discriminante servirà allo scrittore milanese per stroncare la poesia di Prati, adducendo, fra le righe, che «fra tutti i generi della poesia, la lirica è la più alta e la men pieghevole agli ingegni» (Rovani 1874a: 110). Il saggio su Prati, inoltre, diventato una breve storia della lirica italiana, ci illumina su un aspetto essenziale: per Rovani la lirica non è adatta a rappresentare il «pensiero collettivo», compito che, naturalmente, sarà raccolto dal romanzo, ma anche da altre forme espressive meno compromesse dalla visione autoreferenziale della voce lirica.

Nello stesso modo, il capitolo dedicato a Giusti serve a Rovani per introdurre la storia della satira e serve a noi per capire quale ruolo essa compirà all'interno del sistema letterario milanese. Da una parte, la satira contagia interamente una cultura'; dall'altra, essa è la forma con la quale Milano più si identifica, perché «antica come la sua storia» (Rovani 1874a: 162). Tali osservazioni gettano un po' di luce sulla sua stessa teoria del romanzo. Se per Rovani, le espressioni artistiche si alimentano reciprocamente perché fanno parte di uno stesso sistema culturale, sarà innegabile che il romanzo lombardo raccolga la deriva ironica, malinconica e dolorosa che la satira imprime. Il discorso sui propositi assolutamente «seri» ${ }^{10}$ dello scrittore satirico è indicativo di quanto Rovani aspirasse con le sue pagine corrosive nei Cento anni non solo una visione disincantata del mondo ma ad un posizionamento identitario inequivocabile all'interno di una tradizione ben consolidata.

\footnotetext{
«Non ebbe genio, ebbe ingegno: fu vastamente versatile, non profondo» (Rovani 1874a: 105).

«La satira è indizio di superiorità mentale di un paese; potrebbe aggiungersi che è indizio della sua vita travagliata ed infelice» (Rovani 1874a: 162).

10 «Il sintomo sincero che accusa il vero poeta satirico è la serietà» (Rovani 1874a: 166).
} 
In poche parole, tutta la narrativa dell'Ottocento ruota attorno al capolavoro manzoniano, senza che ce ne siano stati altri capaci di spodestarlo. Tale tensione si percepisce in ogni pagina dedicata al romanzo. L'inclusione di tanti romanzi sotto l'egida manzoniana, così come la segnalazione di quelli che tentarono nuove vie sembrano essere le strategie critiche che conducono alla riflessione sul ruolo che Rovani stesso riserva per sé nella scacchiera narrativa dell'Italia. Non è casuale che tutte queste riflessioni, praticamente parallele alla prima stesura del romanzo, fungano non da laboratorio pratico di produzione narrativa, ma da fucina teorica da cui, nello stesso tempo, va disegnandosi il romanzo.

\subsection{Concetti e categorie}

Noi che abbiamo letto Il Castello di Trezzo quando eravamo fanciulli e che abbiamo preso tanto interesse alla figura grottesca e misteriosa di Ariele e alla bella armatura di Palamede e alla voce di Ginevra che confortava della nota canzone il dolce amante [...], noi non possiamo adesso farci capaci di quell'entusiasmo che pure ha destato nella nostra tenera intelligenza e nel nostro cuore ancora più tenero (Rovani 1874a: 205).

Le ragioni di un tale entusiasmo sono analizzate puntualmente da Rovani (1874a: 205-206): in quanto «romanzo di divertimento», Il Castello di Trezzo era fatto di accumulazione di avventure sulla base di un intreccio avvincente capace di destare «un'ansietà irresistibile» e condito di una «morale di convenzione che fa morire il malvagio e trionfare l'uomo virtuoso ». A tali ragioni, egli aggiunge una lunga serie di tratti al negativo che caratterizzerebbero le produzioni narrative secondo l'idea e la forma del romanzo di Bazzoni. Proprio questa evidenziazione al negativo delle caratteristiche di tali romanzi fa trasparire quali debbano essere gli elementi costitutivi di un romanzo destinato esso sì a far parte della tradizione: novità, grandezza d'intento, vivacità nella creazione, "dipintura" dei caratteri, elevazione e accuratezza di stile, lingua ricca, eguale e alta (Rovani 1874a: 206).

La riflessione sul romanzo non finisce qui. Due problemi essenziali si incrociano ne Le Tre Arti: il ruolo della storia dopo il discorso di Manzoni sul romanzo storico e il problema del vero. Ma questi due snodi del romanzo - e dell'arte - italiano dell'Ottocento non sono affrontati solo nella prima parte, sugli scritti letterari, ma anche nella seconda e nella terza, sulla musica e sulle arti figurative.

Nella prima parte, all'interno del canone di scrittori illustri, Rovani annovera Antonio Zoncada, i cui lavori storici (a cui Rovani deve anche più di un'informazione per la composizione della propria crestomazia) pongono il problema del metodo storiografico di Guizot. Il metodo di Guizot consisterebbe nel presentare i casi storici così come sono avvenuti per poi, senza teorie precostruite, trarne le conseguenze. Si pensi quanto queste osservazioni spieghino il metodo con cui lo stesso Rovani affrontò più che altro nei Cento anni la questione storica: presentando in generale all'inizio di ogni epoca (si ricordi che il volume è diviso in epoche storiche oltre che in libri) una sintesi degli eventi essenziali, il narratore passa immediatamente dopo alle osservazioni generali a partire dalle quali è possibile leggere gli eventi narrativi che si stanno per raccontare. E non viceversa. La storia irrompe nella vita dei personaggi dei Promessi Sposi, come ha notato Brogi (2018), mentre, a differenza di Manzoni, in Rovani i grandi fatti ci vengono dispiegati all'inizio, in modo che noi possia- 
mo trarne le conseguenze. Un cambio radicale di prospettiva storica che illumina senz'altro il processo di costruzione dei Cento anni.

$\mathrm{Ma}$, a dire il vero, a fungere da punto di appoggio essenziale per capire quale sia il ruolo della storia nell'arte, vengono in aiuto, più che le riflessioni sul romanzo, quelle sulla pittura. Dopo aver identificato scuole pittoriche nell'Italia dell'Ottocento (quella fiorentina, quella romana, quella veneziana e quella lombarda) e dopo aver delineato la genealogia di maestri e discepoli, Rovani, pur entusiasta dei quadri di Hayez, si riserva una critica personale:

La pittura storica non consiste già nel preferire alla toga e ai fasci consolari, le corazze e i velluti del Medio Evo. La giacchetta di fustagno e i calzoni a pedule, la cuffia e lo sciallo possono entrare in un quadro storico e non essere per nulla ostacolo allo sviluppo delle grandi idee e degli affetti forti e generatori (Rovani 1874b: $133)$.

Quindi, ogni opera che si degni di essere storica dovrà rinunciare al pittoresco per raggiungere invece, mediante una ricostruzione non necessariamente retrospettiva, la sua propria verità. Vengono in mente, qui, le parole di Bachtin in Epos e romanzo circa la ineliminabile contemporaneità di ogni romanzo moderno, al di là della retrocessione temporale, anche in chiave storica, della trama (Bachtin 1938 [1970]). Non è un caso che i Cento anni tendano a una storia della contemporaneità per evitare $\mathrm{i}$ rischi del falso storico e del pittoresco, rischio già sfiorato da Rovani nei primi romanzi degli anni ' 40.

Fondamentale, infine, la distinzione tra storia civile e storia domestica, cui attinse l'Arienti nelle sue opere. Per Rovani (1874b: 148), la pittura di Carlo Arienti si muove armonicamente tra due poli non facilmente conciliabili: quello della storia civile, appresa dai libri e poi tradotta in arte «ad ammaestramento delle moltitudini» e, invece, quello della storia domestica, appresa dall'esperienza, e che desta «la riflessione degli uomini pensatori». Quindi, due tipologie destinate a due lettori o spettatori diversi. Chissà a chi pensava Rovani ogni volta che costruiva la sua scena domestica o quando, ad esempio, si vide condotto a rappresentare un evento traumatico come la morte di Prina?

Passiamo ora al problema del vero. In una nota del suo saggio su Rovani, Baldi segnala che 1'autore non fece mai alcuna menzione a Balzac. Invece, nell'analisi sull'opera di Guerrazzi, che Rovani (1874a: 226) trova piena di meriti, si afferma che «Balzac non era ancora celebre allora [cioè con la pubblicazione de La battaglia di Benevento], e l'anatomia profonda del cuore umano Guerrazzi l'aveva già tentata con pari verità del Francese.». Il brano sembra superficiale, ma racchiude in sé nientemeno che il problema della verità, concetto davvero complessissimo e inerente tutta la narrativa dell'Ottocento. A tal punto che, forse, in Italia servirebbe un volume intero solo sulla questione del vero, studiato caso per caso nelle varie declinazioni dei narratori e dei poeti.

Nella lunga disquisizione dell'apporto del Manzoni nell'ambito, non già della letteratura, ma del pensiero filosofico, politico e sociale, nonché religioso, o addirittura, secondo Rovani, teologico, analizzando la disputa contro Bentham nella Morale cattolica, Rovani (1847a: 49) si sofferma sul metodo manzoniano: «questo discorso di Manzoni deve essere letto e studiato in tutta la sua integrità da chi vuole sentire immediatamente gli effetti di quella dialettica prodigiosa che piega e doma il pensie- 
ro, e lo sforza a genuflettersi, per così dire, innanzi all'altare della verità». La verità, però, non è un dogma né un'ideologia, non sarà neanche, come per molti dei suoi coetanei, capacità scientifica di rappresentazione della realtà: la verità è in Rovani un principio cardine che solo l'artista raggiunge in un gioco insondabile di intuizioni e fatica fisica, spontanea ed epifanica rivelazione e ricerca materiale. Nella terza parte del suo trattato, occupandosi della colonna celebrativa di Austerlitz, realizzata da Bartolini a Place Vendôme, Rovani (1874b: 93) la giudica, in quanto critico d'arte, «piena di storica verità», in cui verità significa «verità poetica e di slancio e di fremito». La verità, pertanto, è frutto della fantasia, perché «non è artista se non chi inventa» (Rovani 1874b: 97), cosicché, analizzando il Monumento di Leon Battista Alber$t i$ dello stesso Bartolini, Rovani esprime, quasi commosso, che nella sua esecuzione «è verità scrupolosa, non gretto realismo» (1874b: 89).

La verità, comunque, può essere rappresentata. Alla puntuale disamina dell'opera scultorica di Luigi Duprez, Rovani oppone un rapido accenno alle opere di Giuseppe Pierotti, allora noto scultore, oggi pressoché dimenticato. Pierotti avrebbe l'attitudine a riprodurre il vero, ma non «d'interrogare questo vero, cogliendone quelle bellezze che non si rivelano che al senso estetico» (Rovani 1874b: 198).

Verità è la stessa parola che Rovani assegna in modo assoluto alla luce che la Francia ha dato alla cultura nell' Ottocento ${ }^{11}$. Non è paradossale o contraddittorio rispetto a quanto è stato detto circa l'influenza obliqua e non diretta del pensiero francese su quello italiano. Il punto è che un discorso su un qualsiasi argomento che tenti di addentrarsi in questioni di un certo spessore va costruito - secondo Rovani - entro una serrata logica del pensiero e dell'esecuzione artistica, in funzione di un unico principio cardine: la verità. Di questa lezione manzoniana - vale a dire, di come Manzoni abbia costruito un percorso complesso che porta alla verità - lo scrittore milanese fa tesoro e tutta la sua opera mira a raggiungere la stessa meta. Sia nelle sue osservazioni critiche, di carattere saggistico, sia nella prosa narrativa intrisa di saggismo, Rovani aspira a dimostrare qualcosa. Poco importa ora cosa. Ciò che importa è rilevare che esiste la consapevolezza assoluta di un metodo. E non, come tanta critica ripete ormai da cento anni a questa parte, un insieme disgregato senza filo né disegno né orizzonte. Appare ovvio che il metodo rovaniano, conclusasi la fase fondativa, unica ed irripetible, non può essere quello manzoniano. Ma ciò che rimane in piedi è l'edificio di principi e presupposti, dai quali non è bene distaccarsi con troppa scioltezza. L'edificio, quindi, è sorretto sia dalla convinzione del primato della storia, intesa come fondamento di un qualsiasi approccio alla cultura; sia dalla ricerca di un "assunto" - per dirla con Rovani - che implichi davvero un'innovazione in quel campo della cultura e apporti un'innovazione al sistema in movimento e che non tradisca la verità che solo l'artista coglie.

Infine, uno degli ultimi concetti più interessanti nella teoria estetica rovaniana attorno al romanzo è quello di pubblico. Debitore del clima dell'epoca, che già dalla fine del Settecento aveva assegnato al lettore o allo spettatore il ruolo di arbitro del gusto, il suo ragionamento però si distacca fortemente da chi pretende attribuire all'approvazione di tale pubblico, anche massiva, una valutazione estetica pertinente o duratura.

11 Cfr. il secondo capitolo del Libro XX, dedicato alla venerazione di Parigi, come "capitale del mondo" (Rovani 2008: 1126-1128). 
Il pubblico è come una bell'amante; per conquistarla non sono mai troppi i sagrifizi e le proteste e le divozioni; ma, conquistata che sia, giovano persino le infedeltà e gli strapazzi per mantenerla serva devota. [...] Se non che, anche il pubblico, come le amanti tenute schiave dagli arbitri amatori, si ricatta alla prima occasione, e si riserba il diritto di rappresaglia per quelle occasioni precisamente in cui gli amatori bisbetici ritornano ai primi entusiasmi (Rovani 1874b: 20).

Abbiamo già detto che la stessa idea di lettore implicito nel testo (o lettore modello, come lo chiamò Umberto Eco) è uno degli elementi che distanziano Rovani da Manzoni. Ma l'idea di pubblico come depositario capriccioso della verità ultima del testo, infedele e inaffidabile, non solo lo allontana dal ruolo paternalistico di Manzoni scrittore, bensì lo lancia con impeto verso l'irriverenza scapigliata, che tanto deve all'autore dei Cento anni.

\section{Per finire}

L'ultimo capitolo del suo lungo discorso critico riguarda Domenico Induno, l'analisi delle cui opere permette a Rovani di entrare in dibattito con Selvatico circa la pittura di genere ${ }^{12}$. A noi, però, preme segnalare che se la prima parte de Le Tre Arti inizia con Manzoni, questo capitolo dell'ultima parte si chiude di nuovo con Manzoni, dopo cinquecento pagine di idee, posizioni, teorie, sentenze e giudizi critici. Il discorso è sinteticamente il seguente: non è accettabile la condanna della pittura di genere da parte di Selvatico nei confronti della pittura storica. Perché se Manzoni e prima di lui Scott, avendo composto dei romanzi storici, sono stati capaci di «aver riprodotti i grandi avvenimenti di epoche tramontate», non fu minore l'importanza di tutta quella letteratura che introdusse il lettore nell'universo contemporaneo e li «condusse attraverso i segreti, alle miserie e alle piaghe della nostra vita» (Rovani 1874b: 232). «Abbiamo bisogno - sostiene Rovani (1874b: 233) in chiave programmatica, proprio mentre aveva già iniziato a pubblicare a puntate i Cento anni - di essere introdotti ad assistere ai gravi dolori nascosti nelle famiglie, ai quei dolori che più o meno direttamente s'annettono alla vita pubblica». Ma non in chiave realistica (aderendo all'estetica del brutto come in Victor Hugo) né secondo il «funesto naturalismo» in voga. Il come lo si vedrà nei Cento anni. Rovani, appresa e assimilata la lezione manzoniana, considerata da egli stesso compiuta, ha bisogno invece di raggiungere a modo suo un'altra verità, una nuova verità.

\section{Riferimenti bibliografici}

Albertazzi, Adolfo (1909): Ora e sempre: romanzo, Milano, Treves.

Alfano, Giancarlo / De Cristofaro, Francesco (2018): Il romanzo in Italia. L'Ottocento, Roma, Carocci.

12 A dire la verità, non è l'ultimo capitolo dell'edizione originale del IV volume della Storia delle lettere, poi ripubblicato come Biografie (1865). Si tratta del terzultimo, ma che, in realtà, nell'edizione postuma del 1874 , funge effettivamente da conclusione, anziché i due capitoletti finali del 1858, dedicati a Sogni, Vitale, Bellosio e Casnedi e alla scuola veneta. 
Bachtin, Michail (1938[1970]): «Epos e romanzo», Estetica del romanzo, Torino, Einaudi, pp. 445-482.

Baldi, Guido (1967): Giuseppe Rovani e il problema del romanzo nell'Ottocento, Firenze, Olschki.

Borlenghi, Aldo (1961): «Introduzione», in Narratori dell'Ottocento e del Primo Novecento, a cura di A. Borlenghi vol. I, Milano/Napoli, Ricciardi, pp. i-xxxix.

Brogi, Daniela (2018): Un romanzo per gli occhi. Manzoni, Caravaggio e la fabbrica del realismo, Roma, Carocci.

Croce, Benedetto (1914): La letteratura della Nuova Italia. Saggi critici, vol. I, Bari, Laterza.

Dossi, Carlo (1947): Rovaniana, a cura di G. Nicodemi, Milano, Vinciana.

Eco, Umberto (1979): Lector in fabula. La cooperazione interpretativa nei testi narrativi, Milano, Bompiani.

Gutierrez, Beniamino (1934): «La tragedia spirituale di Rovani», in G. Rovani, Cento anni, Milano, Rizzoli, vol. I, pp. 3-16.

Mariani, Gaetano (1967): Storia della Scapigliatura, Caltanissetta/Roma, Salvatore Sciascia Editore.

Mazzoni, Guido (1938): L'Ottocento, parte prima, prima ristampa corretta e aggiornata, Milano, Vallardi.

Nardi, Piero (1923[1968]): La Scapigliatura. Da Giuseppe Rovani a Carlo Dossi, Zanichelli, Bologna. [Seconda edizione accresciuta, Arnaldo Mondadori, Milano].

Portinari, Folco (1970): «Narrativa tra idillio e rivolta», in F. Portinari (ed.), Narratori settentrionali dell'Ottocento, Torino, UTET, pp. 9-75.

Portinari, Folco (2008): «I Cento anni ovvero la crisi del romanzo italiano dopo i Promessi Sposi», in G. Rovani, Cento anni, Torino, Einaudi, pp. vii-xxvi.

Romanò, Angelo (1958): Il secondo romanticismo lombardo e altri saggi sull'Ottocento italiano, Milano, Fabbri.

Rovani, Giuseppe (1843): Lamberto Malatesta, Milano, Libreria Ferrario.

Rovani, Giuseppe (1844): Valenzia Candiano, Milano, Tipografia Guglielmini e Libreria Ferrario.

Rovani, Giuseppe (1845-1846): Manfredo Pallavicino o i Francesi e gli Sforzeschi. Storia italiana, 4 voll., Milano, Borroni \& Scotti.

Rovani, Giuseppe (a cura di) (1855-1858): Storia delle Lettere e delle Arti giusta le reciproche loro rispondenze, ordinata nelle vite e nei ritratti degli uomini illustri dal XIII secolo ai nostri giorni, 4 voll., Milano, vol. I-II, Borroni \& Scotti; vol. III-IV, Sanvito-Borroni \& Scotti.

Rovani, Giuseppe (1859-1864): Cento anni. Romanzo ciclico, Milano, vol. I-III, Tipografia Wilmant; vol. IV-V, G. Daelli e C.

Rovani, Giuseppe (1868-1869): Cento anni. Romanzo ciclico, seconda edizione riveduta ed illustrata, Milano, Stabilimento Redaelli dei Fratelli Richiedei.

Rovani, Giuseppe (1868): La Libia d'oro. Scene storico-politiche, Milano, Stabilimento Redaelli della Società Chiusi e Richiedei.

Rovani, Giuseppe (1872): La giovinezza di Giulio Cesare, Milano, Legros.

Rovani, Giuseppe (1874a): Le Tre Arti considerate in alcuni illustri italiani contemporanei, Milano, Treves. vol. I (copia anastatatica: Milano, Lampi di Stampa, 2005).

Rovani, Giuseppe (1874b): Le Tre Arti considerate in alcuni illustri italiani contemporanei, Milano, Treves, vol. II (copia anastatatica: Milano, Lampi di Stampa, 2005).

Rovani, Giuseppe (2008): Cento anni. edizione rivista e corretta, introduzione di F. Portonari, nota al testo di M. Giachino, Torino, Einaudi. 
Scrima, Valentino (2004): Giuseppe Rovani critico d'arte, Milano, LED.

Tamiozzo Goldmann, Silvana (1994): Lo Scapigliato in Archivio. Sulla narrativa di Giuseppe Rovani, Milano, Francoangeli.

Tamiozzo Goldmann, Silvana (2018): «La vocazione della Storia e l'opera di Rovani», in G. Alfano, F. De Cristofaro (eds.), Il romanzo in Italia, vol. II: L'Ottocento, Roma, Carocci, pp. 137-148.

Tellini, Gino (1998): Il romanzo italiano dell'Ottocento e del Novecento, Milano, Bruno Mondadori.

Zoncada, Alberto (1853): I Fasti delle Lettere in Italia nel corrente secolo additati alla studiosa gioventù, Milano, Gnocchi. 